\title{
Geometric phase in weak measurements
}

\author{
Erik Sjöqvist* \\ Department of Quantum Chemistry, Uppsala University, Box 518, Se-751 20 Uppsala, Sweden
}

(Dated: August 29, 2018)

\begin{abstract}
Pancharatnam's geometric phase is associated with the phase of a complex-valued weak value arising in a certain type of weak measurement in pre- and post-selected quantum ensembles. This makes it possible to test the nontransitive nature of the relative phase in quantum mechanics, in the weak measurement scenario.
\end{abstract}

PACS numbers: 03.65.Vf, 03.65.Ta

Pancharatnam's 1] notion of 'in-phase' is the underlying principle of the quantal geometric phase [2, 3]. It is based on the physical condition of maximal interference: the vector representatives $|A\rangle$ and $|B\rangle$ of two nonorthogonal quantal states $A$ and $B$ are in-phase if they produce a maximum in intensity when added. This amounts to the condition $\langle B \mid A\rangle>0$, and any deviation from it defines the Pancharatnam relative phase $\phi_{B A}$ as

$$
e^{i \phi_{B A}}=\frac{\langle B \mid A\rangle}{|\langle B \mid A\rangle|},
$$

i.e., the phase of the complex-valued scalar product $\langle B \mid A\rangle$.

The Pancharatnam relative phase is a nontransitive concept in the sense that it is possible to have $\phi_{B A} \neq 0$ although both $\phi_{C B}$ and $\phi_{A C}$ vanish. Moreover, if $\phi_{C B}=$ $\phi_{A C}=0$ then the excess phase $\phi_{B A}$ equals the geometric phase

$$
\Delta(A, B, C)=\arg \langle A \mid C\rangle\langle C \mid B\rangle\langle B \mid A\rangle
$$

for the geodesic triangle connecting the quantal states $A, B$, and $C$ in projective Hilbert space. For a qubit, such as the polarisation of a photon, $\Delta(A, B, C)$ is proportional to the solid angle of the spherical triangle on the Bloch sphere with vertices at $A, B$, and $C$.

The triangle phase $\Delta$ is the smallest nontrivial entity of the geometric phase. To see this, let $A_{1}, A_{2}, \ldots, A_{n}$ be an arbitrary sequence $c$ of pure quantal states. The geometric phase $\gamma[c]$ associated with this sequence takes the form

$$
\gamma[c]=\arg \left\langle A_{1} \mid A_{n}\right\rangle\left\langle A_{n} \mid A_{n-1}\right\rangle \ldots\left\langle A_{2} \mid A_{1}\right\rangle .
$$

Using elementary properties of complex numbers, one may rewrite $\gamma[c]$ as 4$]$

$$
\gamma[c]=\sum_{k=2}^{n-1} \Delta\left(A_{1}, A_{k}, A_{k+1}\right) .
$$

In the continuous limit, this takes the form

$$
\gamma[c]=\int_{0}^{T} \Delta\left(A_{0}, A_{t}, A_{t+d t}\right)
$$

\footnotetext{
*Electronic address: eriks@kvac.uu.se
}

$$
=\arg \left\langle A_{0} \mid A_{T}\right\rangle+i \int_{0}^{T}\left\langle A_{t} \mid \dot{A}_{t}\right\rangle d t,
$$

where $t \in[0, T] \rightarrow A_{s}$ is a smooth path in projective Hilbert space. If $\left|A_{0}\right\rangle$ and $\left|A_{T}\right\rangle$ are in-phase, then $\gamma[c]$ reduces to the definition of geometric phase in $[5,6]$.

Here, we propose a quantitative test of the nontransitive nature of Pancharatnam's concept of relative phase by utilising weak measurements 7]. It measures $\Delta(A, B, C)$ in terms of the phase of a complex-valued weak value 7] obtained in a weak measurement of a onedimensional projector performed between two complete projective measurements.

The standard techniques to measure the geometric phase are based on interferometric 8] or polarimetric ideas 9]. Common to these ideas is their need for phase calibration, which constitutes an extra constraint in the experimental set up. Curiously, as shall be clear from the following analysis, phase calibration seems not needed in the weak measurement scenario.

Let us first delineate in what sense polarimetry and interferometry involve phase calibration. In polarimetry, an additional variable $\mathrm{U}(1)$ shift $e^{i \chi}$ is applied to $|A\rangle$ or $|B\rangle$, when superposed. The resulting state is thereafter projected onto the third state $C$, yielding the intensity

$$
\mathcal{I}_{p} \propto 1+\operatorname{Re}\left(e^{-i \chi}\langle A \mid C\rangle\langle C \mid B\rangle\right)
$$

where we have assumed that $A$ and $B$ have equal probability weight and that $e^{i \chi}$ is applied to $|A\rangle$. The shift in the interference oscillations equals $\Delta(A, B, C)$ provided $\chi$ is set to zero at $\phi_{A B}$. Thus, to measure the geometric phase we must first obtain the relative phase between $|A\rangle$ and $|B\rangle$, e.g., by measuring the intensity ||$A\rangle+\left.|B\rangle\right|^{2} \propto 1+|\langle A \mid B\rangle| \cos \phi_{A B}$, and thereafter shift the interference pattern in Eq. (6) by $\phi_{A B}$.

A more symmetric expression than Eq. (6) is obtained in interferometry, where an input internal state $A$ is distributed on two spatial particle beams. One of the beams is exposed to a sequence of projections $|A\rangle \rightarrow|B\rangle\langle B \mid A\rangle \rightarrow|C\rangle\langle C \mid B\rangle\langle B \mid A\rangle$, while the other beam is exposed to the variable $\mathrm{U}(1)$ phase shift $e^{i \chi}$. The resulting intensity oscillations

$$
\mathcal{I}_{i} \propto 1+\operatorname{Re}\left(e^{-i \chi}\langle A \mid C\rangle\langle C \mid B\rangle\langle B \mid A\rangle\right)
$$

are shifted by the geometric phase $\Delta(A, B, C)$ provided $\chi$ is kept to zero relative a reference interferometer defined 
by removing the projections. Physically, this means that the relative path length of the two interferometer arms are kept equal throughout the experimental run.

Now, we describe the weak measurement approach to measure the geometric phase $\Delta(A, B, C)$. Let a quantum system and measurement device be prepared in the product state

$$
\varrho_{0}=|A\rangle\left\langle A|\otimes| M_{0}\right\rangle\left\langle M_{0}\right| .
$$

We take the initial apparatus wave function in the position representation as a Gaussian. Explicitly, we may take $M_{0}(q)=\left\langle q \mid M_{0}\right\rangle \sim e^{-q^{2} /\left(2 \sigma^{2}\right)}$, where $|q\rangle$ is an eigenvector of the pointer position operator $Q$. The system and device are made to interact during a finite time interval as described by the Hamiltonian 10

$$
H(t)=g(t) \mathcal{P}^{B} \otimes Q .
$$

Here, $\mathcal{P}^{B}$ is assumed to be a one-dimensional projector, i.e., $\mathcal{P}^{B}=|B\rangle\langle B|$, and the time-dependent coupling parameter $g$ turns on and off the interaction between the measurement device and the measured system. When the interaction is over, we obtain the total state

$$
\varrho=e^{-i \kappa \mathcal{P}^{B} \otimes Q}|A\rangle\left\langle A|\otimes| M_{0}\right\rangle\left\langle M_{0}\right| e^{i \kappa \mathcal{P}^{B} \otimes Q}
$$

with the measurement strength

$$
\kappa=\frac{1}{\hbar} \int g(t) d t,
$$

where $2 \pi \hbar$ is Planck's constant. Conditioned on the postselection of the state $|C\rangle\langle C|$, we find

$$
\begin{aligned}
\varrho_{p s}= & |C\rangle\left\langle C\left|\otimes\left\langle C\left|e^{-i \kappa \mathcal{P}^{B} \otimes Q}\right| A\right\rangle\right| M_{0}\right\rangle \\
& \times\left\langle M_{0}\right|\left\langle A\left|e^{i \kappa \mathcal{P}^{B} \otimes Q}\right| C\right\rangle .
\end{aligned}
$$

Now, if $\kappa \sigma \ll 1$ then terms in the order of $(\kappa Q)^{n}, n \geq 2$, can be neglected. Under this condition and the assumption that $\langle A \mid C\rangle \neq 0$, we obtain

$$
\left\langle C\left|e^{-i \kappa \mathcal{P}^{B} \otimes Q}\right| A\right\rangle \approx\langle C \mid A\rangle e^{-i \kappa \mathcal{P}_{w}^{B}(A, C) Q},
$$

where

$$
\mathcal{P}_{w}^{B}(A, C)=\frac{\left\langle C\left|\mathcal{P}^{B}\right| A\right\rangle}{\langle C \mid A\rangle}=\frac{\langle C \mid B\rangle\langle B \mid A\rangle}{\langle C \mid A\rangle}
$$

is the weak value of the operator $\mathcal{P}^{B}$ with respect to the pre-selected state $A$ and the post-selected state $C$. The resulting wave packet of the measuring device reads

$$
\begin{gathered}
M(q) \sim \exp \left(-\frac{\left(q-\kappa \sigma^{2} \operatorname{Im} \mathcal{P}_{w}^{B}(A, C)\right)^{2}}{2 \sigma^{2}}\right) \\
\times \exp \left(-i \kappa \operatorname{Re} \mathcal{P}_{w}^{B}(A, C) q\right) .
\end{gathered}
$$

Thus, as a result of the post-selection, the weak measurement shifts the position of the pointer by

$$
\delta q=\kappa \sigma^{2} \operatorname{Im} \mathcal{P}_{w}^{B}(A, C)
$$

and its momentum by

$$
\delta p=-\hbar \kappa \operatorname{Re}_{w}^{B}(A, C) .
$$

Upon multiplication and division of the right-hand side of Eq. (14) by $\langle A \mid C\rangle$, we obtain the relation

$$
\arg \mathcal{P}_{w}^{B}(A, C)=\Delta(A, B, C)
$$

which, together with Eqs. (16) and (17), yields the main result

$$
\Delta(A, B, C)=-\arctan \left(\frac{\hbar}{\sigma^{2}} \frac{\delta q}{\delta p}\right) .
$$

Thus, by measuring the shift in position and momentum of the measuring device immediately after turning off the weak interaction, we obtain the Pancharatnam geometric phase. Clearly, this deduction is independent of any phase relation between the pre- and post-selected vectors $|A\rangle$ and $|C\rangle$.

The above scheme can be used to measure the geometric phase for any polygon-shaped path $A_{1}, A_{2} \ldots A_{n}$ in projective Hilbert space. Prepare $A_{1}$, measure $\mathcal{P}^{A_{k}}=$ $\left|A_{k}\right\rangle\left\langle A_{k}\right|$ weakly, and post-select $A_{k+1}$. Adding the phases of the resulting weak values $\mathcal{P}_{w}^{A_{k}}\left(A_{1}, A_{k+1}\right), k=$ $2, \ldots, n-1$, yields $\Delta\left(A_{1}, A_{2}, \ldots, A_{n}\right)$.

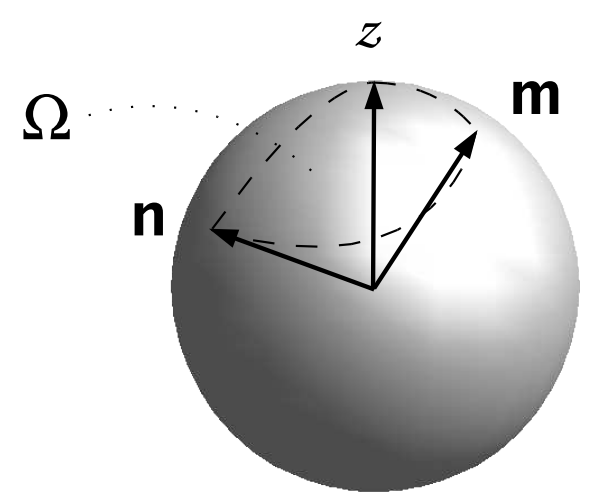

FIG. 1: Bloch sphere representation of weak spin measurement that yields the geometric phase. $\mathbf{n}$ and $\mathbf{m}$ are the preand post-selected states. The solid angle $\Omega$ can be inferred by intermediate weak measurement of the spin in the $z$ direction.

A physically reasonable scenario for the weak measurement procedure could be the following variant of the Stern-Gerlach experiment in [11]. Let the incoming beam of spin- $\frac{1}{2}$ particles be fully polarised in a direction represented by the unit vector $\mathbf{n}$ in three-dimensional space. The spin is projected weakly onto the $\uparrow_{z}$ state, followed by post-selection in a direction represented by another unit vector $\mathbf{m}$. The resulting weak value becomes

$$
\uparrow_{z ; w}=\frac{\sqrt{\left(1+n_{z}+m_{z}+\mathbf{n} \cdot \mathbf{m}\right)^{2}+\left([\mathbf{n} \times \mathbf{m}]_{z}\right)^{2}}}{2(1+\mathbf{n} \cdot \mathbf{m})}
$$




$$
\times \exp \left(-i \frac{1}{2} \Omega\right)
$$

where $\Omega$ is the solid angle of the geodesic triangle on the Bloch sphere with vertices at $\mathbf{n}, z$, and $\mathbf{m}$, see Fig. 1 It follows that $\arg \uparrow_{z ; w}=-\frac{1}{2} \Omega$, which is the expected geometric phase for the geodesic triangle [1, 2, 3].

As is apparent from Eq. (20), the weak value $\uparrow_{z ; w}$ diverges when $\mathbf{m} \rightarrow-\mathbf{n}$. The reason for this behavior is that for a system pre-selected at the point $\mathbf{n}$, there is, in the weak measurement limit, a vanishing probability for it to pass a post-selection of $-\mathbf{n} 12$. Now, the antipodal points $\pm \mathbf{n}$ precisely correspond to orthogonal states for which the geometric phase is undefined. In other words, the case where the geometric phase becomes undefined corresponds to a post-selected quantum ensemble whose size tend to zero in the weak measurement limit.

In conclusion, we have put forward a procedure for measuring Pancharatnam's geometric phase in certain weak measurements in pre- and post-selected quantum ensembles. The proposed test avoids any need for a phase relation between the pre- and post-selected states. Implementation of the weak measurement scheme for observing the Pancharatnam geometric phase should be feasible for neutron spin [13, 14] or photon polarisation [15, 16].

Financial support from the Swedish Research Council is acknowledged.
[1] S. Pancharatnam, Proc. Indian Acad. Sci. A 44 (1956) 247.

[2] S. Ramaseshan and R. Nityananda, Current Sci. 55 (1986) 1225.

[3] M.V. Berry, J. Mod. Opt. 34 (1987) 1401.

[4] N. Mukunda and R. Simon, Ann. Phys. (N.Y.) 228 (1993) 205.

[5] Y. Aharonov and J. Anandan, Phys. Rev. Lett. 58 (1987) 1593.

[6] J. Samuel and R. Bhandari, Phys. Rev. Lett. 60 (1988) 2339.

[7] Y. Aharonov and L. Vaidman, Phys. Rev. A 41 (1990) 11.

[8] A.G. Wagh and V.C. Rakhecha, Phys. Lett. A 197 (1995) 107.

[9] A.G. Wagh and V.C. Rakhecha, Phys. Lett. A 197 (1995)
112

[10] J. von Neumann, Mathematical foundations of quantum mechanics, (Princeton University Press, NJ, 1955), p. 443.

[11] Y. Aharonov, D.Z. Albert, and L. Vaidman, Phys. Rev. Lett. 60 (1988) 1351.

[12] Close to the antipodal point, the magnitude of the weak value can be very large 11 .

[13] R. Golub and R. Gähler, Phys. Lett. A 136 (1989) 178.

[14] R. Golub and R. Gähler, Nucl. Inst. Meth. Phys. Res. A 284 (1989) 357.

[15] J.M. Knight and L. Vaidman, Phys. Lett. A 143 (1990) 357.

[16] G. J. Pryde, J. L. O'Brien, A. G. White, T. C. Ralph, and H. M. Wiseman, Phys. Rev. Lett. 94 (2005) 220405. 